\title{
EGOFET Gated by a Molecular Electronic Switch: a single device memory cell
}

Vitaliy Parkula, Maria Serena Maglione, Stefano Casalini, Qiaoming Zhang, Pierpaolo Greco, Carlo Augusto Bortolotti, Concepció Rovira, Marta Mas-Torrent, Fabio Biscarini*

This paper is dedicated to Professor Claudio Zannoni in occasion of his $70^{\text {th }}$ birthday.

V. Parkula, Dr. P. Greco

Scriba Nanotecnologie S.r.l., Via di Corticella 183/8, 40128, Italy

E-mail:vparkula@scriba-nanotec.com

V. Parkula, Dr. C. A. Bortolotti, Prof. F. Biscarini

Department of Life Sciences, University of Modena and Reggio Emilia, Via G. Campi 103, 41125, Italy

E-mail: fabio.biscarini@unimore.it

Prof. F. Biscarini

Center for Translational Neurophysiology of Speech and Communication (CTNSC), Istituto Italiano di Tecnologia, Via Fossato di Mortara 17/19, 44121 Ferrara, Italy

E-mail: fabio.biscarini@iit.it

Dr. M. S. Maglione, Dr. S. Casalini, Q. Zhang, Prof. C. Rovira, Dr. M. Mas-Torrent Institut de Ciència de Materials de Barcelona (ICMAB-CSIC) and CIBER-BBN, Campus UAB, 08193, Spain

Dr. S. Casalini’s current affiliation: University of Strasbourg, CNRS, ISIS UMR 7006, 8 allée Gaspard Monge, F-67000 Strasbourg, France.

Keywords: organic electronics; electroactive molecules; switchable devices; self-assembled monolayers; Boolean logic circuits

\section{(Abstract)}

Electrolyte-gated organic field effect transistors (EGOFETs) exploit the transduction of interfacial phenomena, such as biorecognition or redox processes, into detectable changes of electrical response. Here, we show that, beyond sensing applications, EGOFETs may act effectively as memory devices, through the functionalization of the gate electrode with a selfassembly monolayer comprising a switching molecule undergoing a large and persistent change of dipole moment, upon application of a small (0.6 V) programming potential. This first example of a switchable EGOFET device with memory retention is based on a tetrathiafulvalene derivative self-assembled on gold and an aqueous buffer as electrolyte in a microfluidic assembly. Changes of the SAM redox state lead to variations of the gate electrochemical potential and, as a consequence, the EGOFET's threshold voltage undergoes reversible shifts larger than $100 \mathrm{mV}$. The distinctive electrical readout upon different redox 
states opens the possibility of writing and erasing information, thus making the transistor as a single memory cell.

\section{Introduction}

Electrolyte-Gated Organic Field Effect Transistors (EGOFETs) are ideal candidates for the next generation of (bio-)sensors in aqueous media due to their ultra-high sensitivity to minute potential changes induced at the electrolyte/device interfaces. ${ }^{[1,2]}$ The electrolyte yields a large effective capacitance $\left(\approx 10^{1}-10^{2} \mu \mathrm{F} / \mathrm{cm}^{2}\right),{ }^{[3-5]}$ which significantly lowers the operational gate voltage with respect to that of solid-state gated Organic Field Effect Transistors (OFETs). A physico-chemical change of either the gate/electrolyte or the electrolyte/semiconductor interfaces induces a response, which can be phenomenologically ascribed to a change of the effective capacitance or a sizable change of the threshold voltage or both, and amplified into large current modulation. As a result, EGOFETs were shown to successfully detect a variety of relevant biological events, ${ }^{[6]}$ such as DNA immobilization and hybridization, ${ }^{[7]}$ recognition of odorant protein binding, ${ }^{[8]}$ dopamine detection, ${ }^{[9]}$ neural signals and action potentials upon extracellular stimulation ${ }^{[10]}$ and antibody-antigen interactions. ${ }^{[11-14]}$

Organic transistors $^{[15,16]}$ are also key components in logic Boolean circuits, where they have been used as inverters, ${ }^{[17,18]}$ ring oscillators, ${ }^{[19,20]}$ and non-volatile memories. ${ }^{[21-23]}$ Apart from bio-sensing applications, EGOFETs have seldom been explored as basic components in logic architectures. $^{[24,25]}$ Recent propositions ${ }^{[26-29]}$ concern the use of organic transistors in aqueous electrolytes as neuromorphic logic components.

In this work, we present an EGOFET operated as a two-state memory element that is able to withstand repeated write/erase cycles. The memory response has been conferred by exploiting the redox characteristics of an electroactive self-assembled monolayer (SAM) grafted on the gate electrode. The use of electroactive SAMs as active components for memory devices has been widely investigated, ${ }^{[30-33]}$ however, their application in electronic devices is still rare. ${ }^{[34]}$ 
To the best of our knowledge, this is the first report of an EGOFET device with memory retention capabilities. The use of EGOFET as memory devices would provide some significant advantages over largely explored OFETs, in particular with respect to the possibility of working in aqueous environment under low potential $(<1 \mathrm{~V})$ without need of encapsulation: these features make EGOFETs ideal devices to be interfaced to or implanted in living systems.

Our EGOFET layout has two top gate electrodes defined as "working electrode" (WE) and "reference electrode" (RE), respectively. The former is SAM-functionalized with the electroactive molecule and is responsible for tuning the device electrical characteristics, the latter has a three-fold role: i) to serve as a counter-electrode in the redox switching process, ii) to provide an internal control assessment of the stability of the device over time and iii) to unambiguously assign changes in the device response to the modulation of the redox state of the electroactive SAM. We demonstrate that the switching of the SAM redox state is strongly correlated to the change of EGOFET threshold voltage. The process is reversible and robust across several writing/erasing/reading cycles, thus leading to a response mimicking that of a one-bit memory device that can be read through the differential current from the integrated reference electrode, thus proving the relevance of this novel multiple-gate EGOFET.

\section{Results and Discussion}

In Figure 1, the EGOFET architecture is presented. It consists of the following components: i) interdigitated source/drain (S/D) electrodes, ii) thin-film OSC, iii) a microfluidic cell and iv) two top gate electrodes. Figure $1 \mathrm{a}$ and $1 \mathrm{~b}$ show the electrical configuration used for the SAM redox switch and the standard I-V characterization of the EGOFET, respectively.

(Figure 1. Device structure and configuration for SAM switching: a) device architecture with assembled microfluidic chamber on top of the S/D electrodes covered with OSC and integration of two top planar gate gold electrodes (WE functionalized with TTFS 2 SAM, RE bare gold) with the electrical circuit used for the application of redox potential; b) device structure showing an oxidized WE electrode and the electrical circuit used for the 
characterization of the dual gate EGOFET; c) structure of $\mathrm{TTFS}_{2} \mathrm{SAM}$ in reduced and oxidized states; d) assembled device with 3D printed fluidic connector (1/32” outer diameter) for integration with peristaltic or syringe perfusion pumps.)

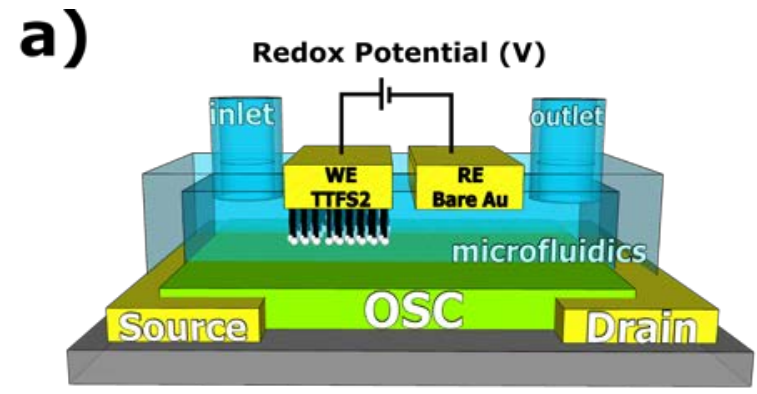

C)

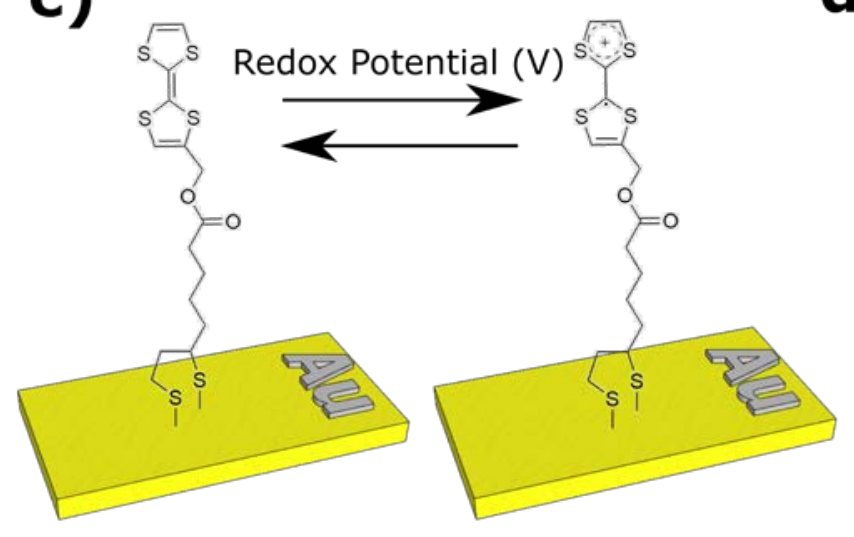

\section{b)}

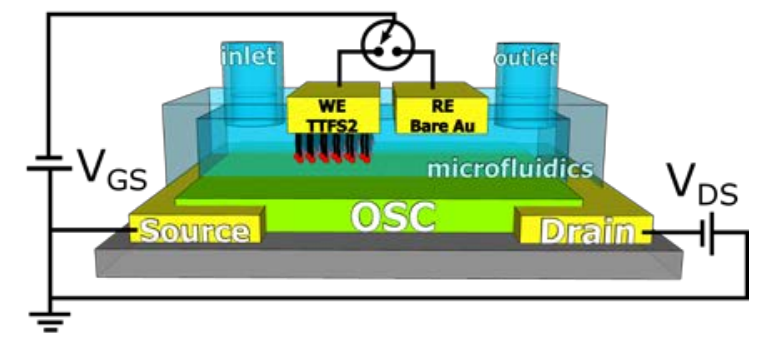

d)

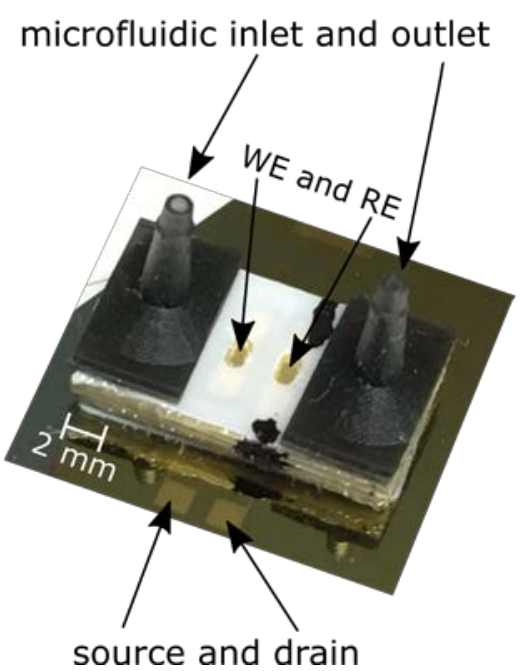

The width-to-length ratio W/L of S/D electrodes is equal to 690. The organic semiconductor (OSC) of choice was Bis(triethylsilylethynyl)-anthradithiophene (diF-TES-ADT), which was deposited blended with polystyrene by means of the "Bar-Assisted Meniscus Shearing" technique previously reported. ${ }^{[35,36]}$ A flexible double-sided adhesive tape was used to create a microfluidics chamber, which was assembled on top of the source and drain (S/D) gold electrodes. The microfluidic cell implemented into the device enables a controlled flux and storage of the aqueous buffer, as well as firmly fixing the two top Au gate electrodes, thereby avoiding any adventitious alteration of wetted gate area. A controlled electrolyte aqueous flux was realized by a peristaltic pump connected via 3D printed connectors. The final assembly of the device is shown in Figure 1d. 
As electroactive switchable SAM, the tetrathiafulvalene derivative [2-2'-bi(1,3dithiolylidene)]-4-ylmethyl5-(1,2-dithiolan-3-yl)pentanoate (TTFS $_{2}$, Figure 1c), that can be easily converted to a stable radical cation, was used. SAMs of $\mathrm{TTFS}_{2}$ were prepared on gold electrodes following the procedure previously reported. ${ }^{[37]}$ Please see the paper by Marchante et al. ${ }^{[37]}$ for thorough characterization of $\mathrm{TTFS}_{2}$ SAMs on gold. Figure S2 reports electrochemical assessment of the TTFS 2 SAM on gold by cyclic voltammetry before device assembly and operation. An aqueous solution of a buffer phosphate $(\mathrm{pH}=6.9)$ and $\mathrm{NaClO}_{4}$ was employed as electrolyte, to ensure the stabilization of the oxidized state of the molecule. As previously mentioned, our EGOFET architecture features two independent planar gold gate electrodes. The WE electrode was functionalized with $\mathrm{TTFS}_{2}$. By applying the proper potential $(+0.6 \mathrm{~V} /-0.6 \mathrm{~V})$ between WE and RE electrodes (Figure 1a), the switch of $\mathrm{TTFS}_{2^{-}}$ based SAM from the reduced (neutral) to the oxidized (charged) state, and vice versa, was achieved. To investigate the influence of the electroactive SAM on the EGOFET response, a differential measurement was carried out by means of recording the response of the device to the gate sweeping of each top gate electrode, then comparing the transfer curves. We will refer to RE-EGOFET, when the EGOFET is operated using the RE electrode as gate contact, and to WE-EGOFET, when the device is gated by WE electrode functionalized by $\mathrm{TTFS}_{2^{-}}$ based SAM. The internal RE gate electrode thus provides a reference I-V characteristics of the device and enables the TTF electrochemical switch without causing any electrical stress to the OSC.

In order to electrically characterize the redox-dependent response of the device, we performed oxidation/reduction cycles of the electroactive SAM as follows: i) I-V recordings of the transfer characteristics of RE-EGOFET and WE-EGOFET; ii) electrochemical oxidation/reduction of $\mathrm{TTFS}_{2}$ SAM by applying a bias equal to $+0.6 \mathrm{~V} /-0.6 \mathrm{~V}$ between WE and RE (where RE is used as the ground upon application of these potentials); iii) I-V recordings of the transfer device characteristics of RE-EGOFET and WE-EGOFET to be 
compared with those recorded at step i). The I-V transfer characteristics of five oxidation/reduction cycles obtained for one device are shown in Figure 2a, where the solid red and blue lines represent the current of the WE-EGOFET featuring $\mathrm{TTFS}_{2}$ in its oxidized and reduced state, respectively, and dashed black lines were used to depict the transfer characteristics of RE-EGOFET.

It is observed that, when the EGOFET is in accumulation mode, the oxidized state exhibits higher drain-source current $\left(I_{D S}\right)$ with respect to the reduced one. This can be understood as the result of oxidation decreasing the electrochemical potential of Au electrode, increasing the work function, ${ }^{[38]}$ and then increasing the voltage drop at the gate/electrolyte interface that is mirrored by a larger voltage drop across the OSC/electrolyte interface (see Figure 2b). ${ }^{[9]}$ This effect leads to a $I_{D S}$ current increase as a consequence of the positive threshold voltage shift of the oxidized WE-EGOFET curves. Such shift is in line with previous reports on the effect of the gate work function on the threshold voltage of EGOFETs. ${ }^{[39,40]}$ Phenomenologically, our redox gated device responds as a two-state ISFET. ${ }^{[41,42]}$

(Figure 2. a) EGOFETs $I-V$ transfer characteristics: Working Electrode within oxidized and reduced states and the Reference Electrode; b) schematic of potential drop at the gate/electrolyte and electrolyte/OSC interfaces reflecting the measured threshold voltage shift.)

a)

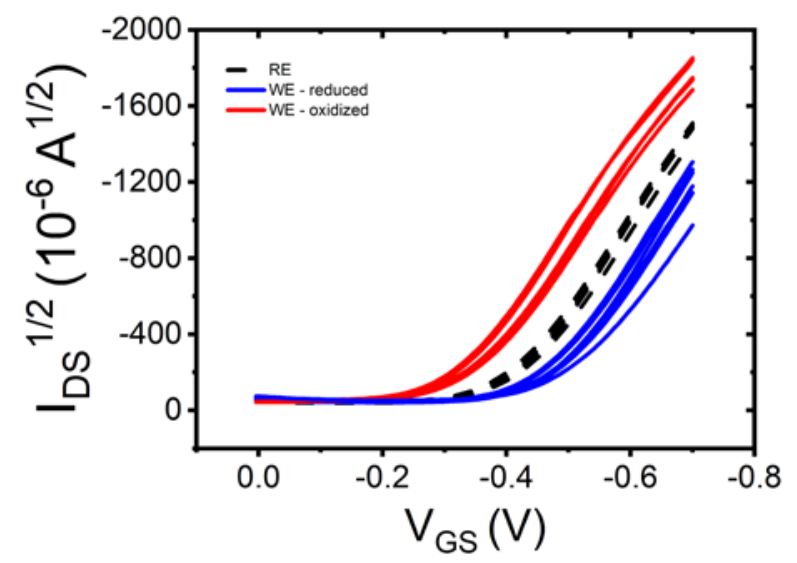

\section{b)}

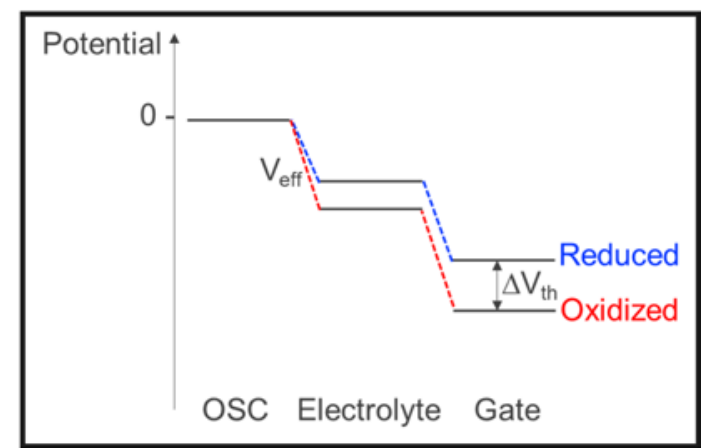

Since EGOFETs are multiparametric devices, different figures of merit might exhibit changes related to the redox state of the SAM headgroup. The behavior of the maximum drain current 
( $\left.I_{D S, M A X}\right)$, the threshold voltage $\left(V_{t h}\right)$ and transconductance $\left(g_{m}\right)$ along five oxidation/reduction cycles of a single device are shown in Figures 3a, 3b and 3c, respectively.

(Figure 3. EGOFET's parameters: a) maximum value of drain-source current, b) threshold voltage and c) transconductance and respective changes upon variation of the electroactive molecule redox state, switching from oxidized $(\mathrm{O})$ to reduced $(\mathrm{R})$.)

a)

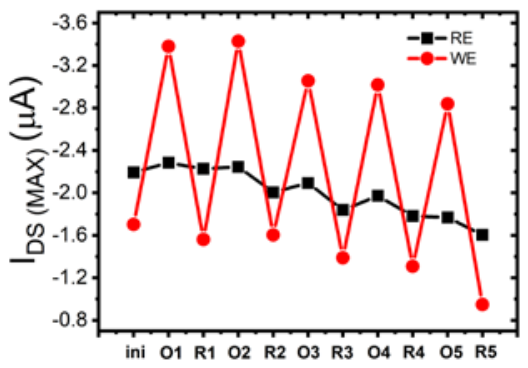

b)

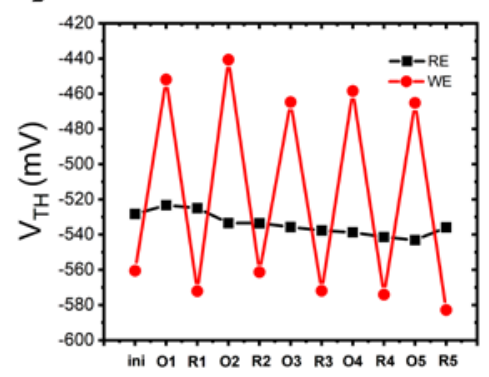

c)

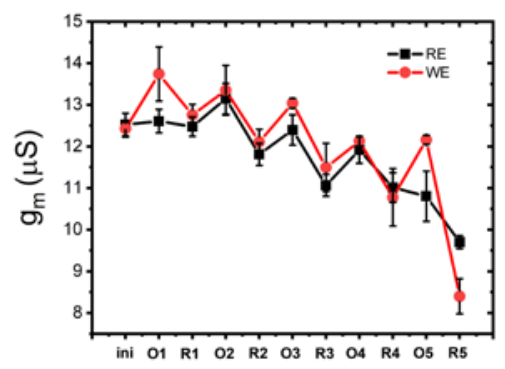

$I_{D S, M A X}$ and $V_{t h}$ appear as the two parameters that are most sensitive to the oxidation state of the SAM, whereas the transconductance $g_{m}$ exhibits overall a marginal difference between the values extracted from either oxidized or reduced WE-EGOFET and the value of the REEGOFET. This evidence hints to the proposition that $I_{D S, M A X}$ and $V_{t h}$ could be good figures of merit to quantitatively assess the presence of either one of the two states (oxidized or reduced) and to validate the stability of the memory effect in WE-EGOFET compared to the REEGOFET. Indeed, at each cycle, the WE-EGOFET $I_{D S, M A X}$ displays changes as high as about $1.5 \mu \mathrm{A}$ and $V_{\text {th }}$ exhibits reversible shifts of about $100 \mathrm{mV}$ for each cycle. At variance with what is observed for the WE-EGOFET, the RE-EGOFET response exhibits much smaller fluctuations that are independent from repeated voltage cycling: in particular, average $I_{D S, M A X}$ and $V_{\text {th }}$ changes are as small as $0.2 \mu \mathrm{A}$ and $3 \mathrm{mV}$, respectively. For this particular device, transconductance $g_{m}$ does not show reversible redox-dependent changes for WE-EGOFET, instead a drift towards smaller values along repeated cycling is apparent. This trend is mirrored by that of RE-EGOFET hinting to the possibility of a bias stress in the device upon repeated redox cycles. 
The low sensitivity of $g_{m}$ to the WE redox state and the highly correlated, redox dependent variations of $I_{D S, M A X}$ and $V_{t h}$ let us infer the working mechanism of the device. The redox reaction between the two different states switches the electrochemical potential of the gate electrode modulating the density of interfacial charges and causing a $V_{t h}$ shift that governs the WE-EGOFET response.

The large, reproducible and reversible variations of $I_{D S, M A X}$ and $V_{t h}$ impart a one-bit memory character to the device: the oxidized state of the $\mathrm{TTFS}_{2}$ on the WE is obtained upon writing, while the reduced state re-emerges upon the erasing of "data". The comparison with the response of RE allows one to address in which state the electronic memory is.

To assess both the robustness and reproducibility of the electrochemically switchable response, we characterized the electrical properties of a total of five different WE-EGOFET devices and measured redox-dependent changes in the transfer characteristics over repeated cycling. The overlay of all the transfer characteristics recorded for distinct devices after each application of an oxidizing potential at the WE is reported in Figure 4a. It is evident, as known in organic electronics devices, that a sizable dispersion is observed when processing is not standardized. This frequent occurrence undermines the meaning of mean values and may discourage one from analyzing the data in a quantitative manner, especially when the relevant parameters are related to derivatives of the experimental curves.

In order to cope with such large dispersion of the transfer curves, we have devised a statistical treatment of the data which is briefly described in the following. Our treatment is based on calculating first an "average" transfer curve for the RE-EGOFET and the WE-EGOFET responses upon oxidation or upon reduction from all the recorded curves (see Figure 4b, where the "average" transfer curve calculated in the case of WE-EGOFET oxidation is reported). Then we extract the parameters from this "average" curve, with their statistical errors. These values of $I_{D S, M A X}, V_{t h}$ and $g_{m}$ are representative for the whole set of transfer curves (from different devices and number of cycles). 
We first took all transfer curves from different devices and cycles for oxidized TTFS 2 and calculated their logarithm as $\ln \left(I_{D S} / \mathrm{A}\right)$. The division of $I_{D S}$ by ampere (A) is necessary to make the argument dimensionless. We then evaluate the logarithmic mean as $\left\langle\ln \left(I_{D S} / A\right)>\right.$, where the brackets indicate an arithmetic mean over the whole data set of transfer characteristics. We estimate the associated error at each $V_{G S}, \delta<\ln \left(I_{D S} / A\right)>$, as the standard error of the mean over the data set. We then calculate what we term the representative transfer curve $<I_{D S}>v S V_{G S}$ as:

$$
<I_{D S}>\cong e^{<\ln \left(I_{D S}(A)>\right.}
$$

A rigorous justification of eq. 1 will be given elsewhere, here suffices to say that it is a widely used approximation for exponentially-varying properties on an ensemble that in graph theory corresponds to the lowest order of a cluster expansion of the exponential mean (often termed “mean field” in the description of the free energy or many-body Hamiltonian).

The associated error $\delta<I_{D S}>$ was estimated by propagating the $\delta<\ln \left(I_{D S} / A\right)>$ as:

$$
\delta<I_{D S}>\cong<I_{D S}>\cdot \delta<\ln \left(I_{D S} / A\right)
$$

The approximation symbol follows from the fact that the representative transfer curve eq. 1 is an approximation to the actual mean transfer curve. The representative transfer characteristics are shown in Figure 4c for TTFS 2 oxidized state (red), reduced state (blue) and for the REEGOFET (black). We notice that the error bars for the RE-EGOFET curves and the reducedWE-EGOFET are much smaller than the one for oxidized WE-EGOFET. The representative curves (continuous lines) of reduced WE-EGOFET and RE-EGOFET are closely matching, indicating that the SAM does not (statistically) alter significantly the device response with respect to the bare Au gate. The three representative curves with their error bars overlap in the off-current region and the onset of the sub-threshold regime $\left(V_{G S}>>-0.4 \mathrm{~V}\right)$ whereas the representative curve for the oxidized state separates from the other two in the saturation and linear regions (a reliable separation at a precise gate voltage must be three times the sum of 
the error bars). This result shows that the most reliable region for readout of the SAM state is the saturation region ( $V_{G S}$ ranging from $-0.3 \mathrm{~V}$ to $-0.5 \mathrm{~V}$ ), even better than the linear region (where smaller differences between $\mathrm{I}_{\mathrm{DS}}$ values can be observed) and especially much better than the sub-threshold region, although this is known to be the most sensitive regime for small variations. The $I_{D S, M A X}$ values are $4.26( \pm 0.33) \mu \mathrm{A}$ and $1.44( \pm 0.04) \mu \mathrm{A}$ for oxidized and reduced WE-EGOFET, and $I_{D S, M A X}=1.72( \pm 0.08) \mu \mathrm{A}$ for RE-EGOFET.

We can also extract $V_{\text {th }}$ and $g_{m}$ from the representative transfer curves for both oxidized and reduced WE-EGOFET. The $V_{t h}$ values are $-0.435( \pm 0.074) \mathrm{V}$ and $-0.559( \pm 0.060) \mathrm{V}$ for the oxidized and reduced state, respectively; $g_{m}$ changes from $17.8( \pm 1.2) \mu S$ in the TTFS 2 oxidized state to $10.1( \pm 0.5) \mu \mathrm{S}$ for the reduced one.

We are now in the position to assess the optimum voltage for readout of the SAM redox state. In consistency with our earlier proposition ${ }^{[14]}$, we define the relative variation of the $I_{D S}$ current of WE-EGOFET as the signal $S=\left[I_{W E}\left(V_{G S}\right)-I_{R E}\left(V_{G S}\right)\right] / I_{R E}\left(V_{G S}\right)$, where $I_{W E}\left(V_{G S}\right)$ and $I_{R E}\left(V_{G S}\right)$ are the transfer curves for WE-EGOFETs and RE-EGOFET, respectively. We replace the signal S from individual curves, as used in ref. [Berto et al.] with the corresponding representative $<\mathrm{S}>$ :

$$
\begin{gathered}
\left\langle S_{o x}\right\rangle \approx \frac{\left\langle I_{\text {ox }-W E}\right\rangle}{\left\langle I_{\text {RE }}\right\rangle}-1 \\
\left\langle S_{\text {red }}\right\rangle \approx \frac{\left\langle I_{\text {red }-W E}\right\rangle}{\left\langle I_{\text {RE }}\right\rangle}-1
\end{gathered}
$$

In Figure $4 \mathrm{~d}$ we plot $<S>$ as a function of $V_{G S}$. There is a peak in $<\mathrm{S}_{\mathrm{ox}}>$ at $V_{G S}=-0.380( \pm$ 0.002) $\mathrm{V}$ (with $<\mathrm{S}_{\mathrm{ox}}>=21.1$ ), whereas $<\mathrm{S}_{\text {red }}>$ takes its maximum value (as low as 0.7 ) at $V_{G S}$ $=-0.156( \pm 0.002) \mathrm{V}$. This confirms that the maximum sensitivity is achieved at $V_{G S}=-0.380$ $( \pm 0.002) \mathrm{V}$. These data show two crucial aspects of this molecular electronic switch: i) the stability of the device response $S$ across repeated cycling and devices and ii) the fact that the value taken by $<S_{o x}>$ at $-0.380 \mathrm{~V}$ in the oxidized state is well distinct from the value of $\left\langle S_{\text {red }}\right\rangle$ 
at the same potential. Then, we infer that $\mathrm{V}_{\mathrm{GS}}=-0.380 \mathrm{~V}$ is the optimum voltage for which the readout of the oxidation state which corresponds to the content of information of the memory. In view of potential memory applications, we have a device whose writing potential is +0.600 $\mathrm{V}$, readout potential is $-0.380 \mathrm{~V}$ and erase potential is $-0.600 \mathrm{~V}$.

(Figure 4. Statistic treatment of $I-V$ transfer characteristics of distinct set of devices: b) "average" transfer characteristic curves with error propagation of a) raw measurements upon application of oxidizing potential at the WE; c) same treatment for the WE reduced and RE demonstrating the favorable region for memory readout, which is justified by d) average signal for oxidized $<S>$ with its peak value at gate-source voltage equal to -0.38 .)

a)
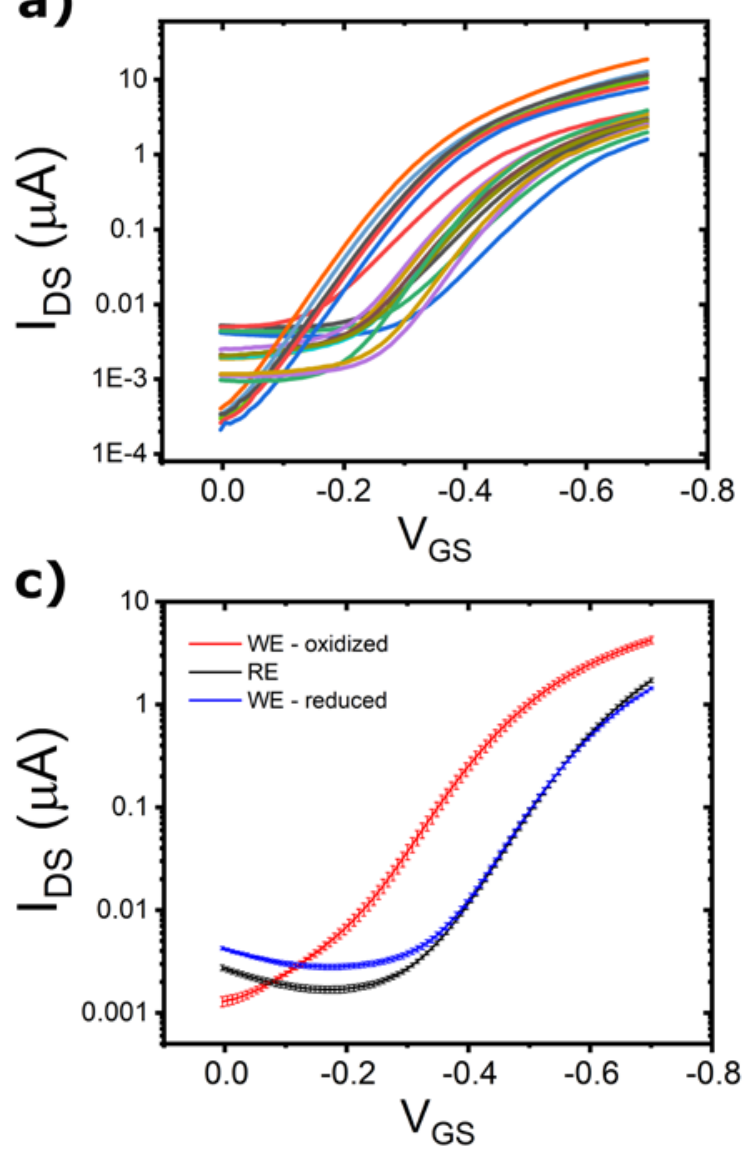

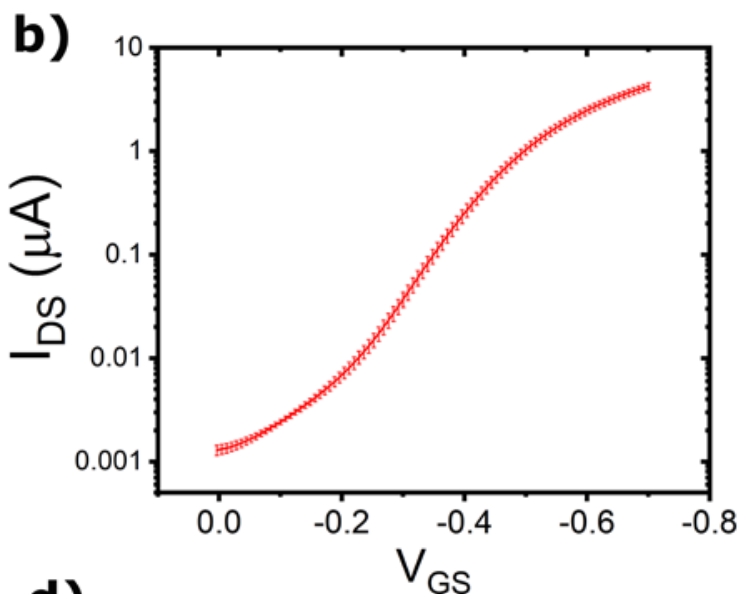

d)

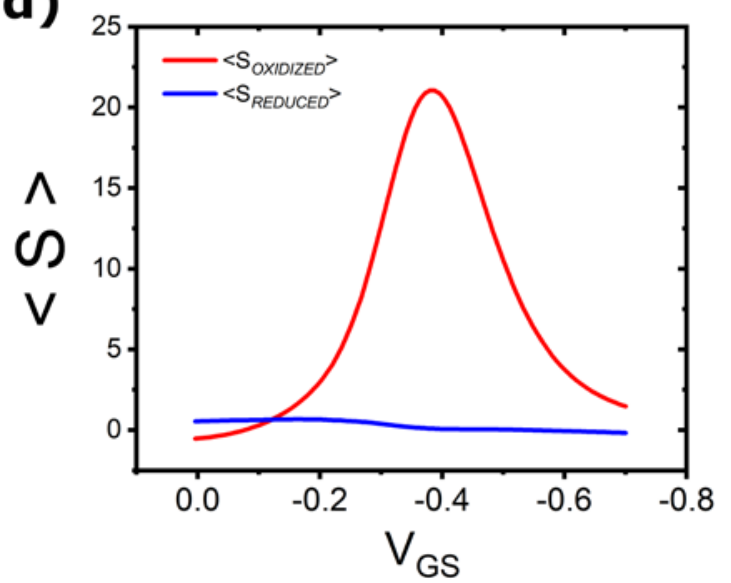

The main potential advantages of EGOFET-based memory architectures with respect to OFETs are the high-current/low-voltage operation to write/read/erase $e^{[21,43-46]}$ and the possibility to work with biological solutions in vitro and potentially in vivo. Nevertheless, one disadvantage is that the EGOFET relies on ion displacement, and this may limit the switching speed of the memory with respect to the OFET. 
In order to assess how volatile the memory is, else what is the retention time of the information, the stability of the oxidized state with time was also explored. It was observed that the written state (oxidized) is retained up to 20 minutes (see Figure 5a) before slowly relaxing back to the erased state. This response indicates that the switch behaves as a "semivolatile” memory. Despite still being too short for practical applications, the retention time exhibited by our device is promising, upon comparison with the recent production of novel memory paradigms in the Organic Bioelectronics field, with typical state retention time in the seconds timescale ${ }^{[47-52]}$ if based on electrochemical doping, although memory effects achieved through charge trapping exhibit retention times up to a few hours. ${ }^{[53,54]}$

Control experiments were carried out in order to verify that the device changes observed were exclusively due to the redox state of the TTF molecule and not to other ionic polarization effects. To this end, an EGOFET device featuring a non-electroactive SAM (viz. 1Decanethiol) replacing the $\mathrm{TTFS}_{2}$-based SAM was fabricated and characterized. The same switching experiments were then performed without observing appreciable changes in the device electrical characteristics upon the application of the write/erase voltages (see Figure 5b). This result unambiguously proves that the redox features of $\mathrm{TTFS}_{2}$ molecules are the sole responsible for the observed electrochemical memory.

(Figure 5. a) extracted threshold voltage values for studying stability of oxidized state during time (red circles) showing memory retention for duration of 20 minutes until it reaches the reduced state (half blue circle); b) control experiment carried with non-electroactive SAM (viz 1-Decanethiol) representing small threshold voltage values shifts to random direction when following the same protocol for oxidation and reduction of WE.) 
a)

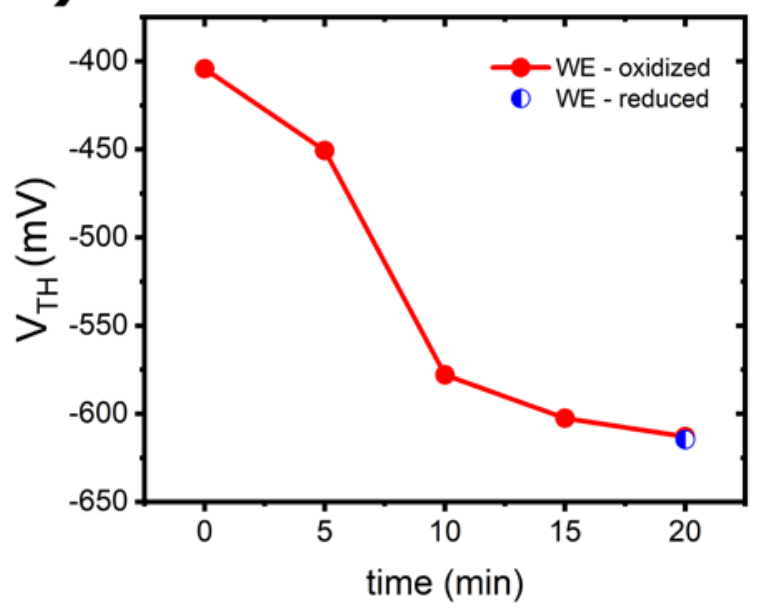

b)

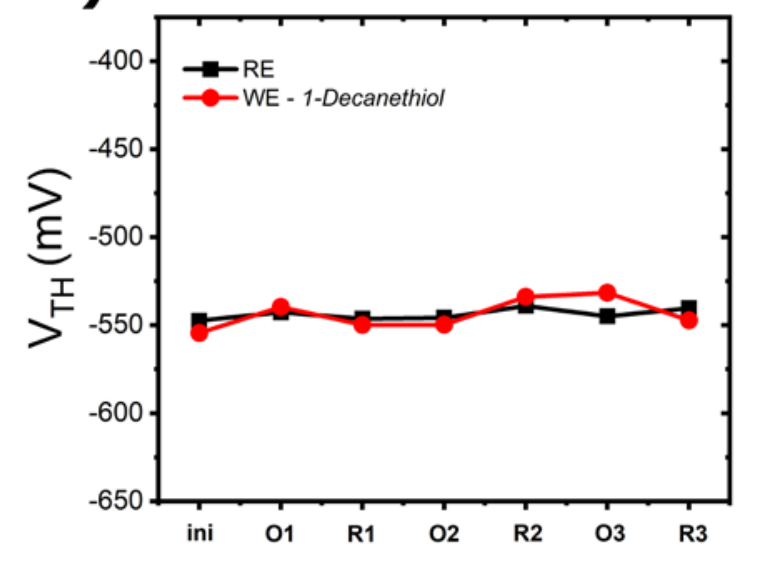




\section{Conclusions}

In conclusion, we have designed and fabricated an externally electrochemically switchable EGOFET employing two gate electrodes: i) a reference RE and ii) a working electrode WE, the latter functionalized with an electroactive TTF-based SAM. The former is exploited as an internal counter-electrode to switch efficiently between the two redox states of the TTF SAM (i.e. oxidized and reduced state). The latter is responsible for driving the device into a readout mode, as the EGOFET response is sensitive to the redox state of the electroactive SAM at the WE in a voltage range corresponding to the saturation regime. The main parameter for readout is $V_{t h}$, which changes by $>100 \mathrm{mV}$ upon switching from oxidized reduced state. Upon global data analysis of the representative transfer curves, we find that transconductance is also altered and that the saturation region is the most reliable for readout of the SAM oxidation state, with the largest $I_{D S}$ changes at $V_{G S}=-0.380 \mathrm{~V}$. Our results show a semi-volatile 1-bit memory element integrated into a dual gate EGOFET, where an electroactive switchable SAM is assembled on one of the gate electrodes. One can envision that a SAM with a molecule withstanding multiple redox states, else a multigate device with different SAMs on each gate electrode, could be a viable route for multi-bit memories integrated into a single electronic device.

\section{Experimental Section}

Gold Source and Drain Electrodes: gold ( $\mathrm{Au})$ source and drain interdigitated electrodes (the channel width and length are $20700 \mu \mathrm{m}$ and $30 \mu \mathrm{m}$ with a geometrical ratio $\mathrm{W} / \mathrm{L}=690$ ) were fabricated on a silicon (Si) substrate. The electrodes layout was photo-lithographically patterned by means of a micro-writer (MicroWriter $\mathrm{ML}^{\mathrm{TM}}$ ) and the metal evaporation was achieved by thermal evaporation (system Auto 306 from Boc Edwards). These electrodes consist of chromium (Cr)/ $\mathrm{Au}(5 \mathrm{~nm}$ and $40 \mathrm{~nm}$, respectively), wherein $\mathrm{Cr}$ is the adhesive layer. 
Gold Gate Electrodes: gate electrodes were obtained from a 4" Si wafer (with a thickness of $525 \pm 25 \mu \mathrm{m}$, single side polished), which were cut into $4 \mathrm{~mm} \times 1 \mathrm{~mm}$ Si dyes. The silicon is heavily As-doped (n-type), with a resistivity ranging from 1 to $6 \mathrm{~m} \Omega \cdot \mathrm{cm}$. A $50 \mathrm{~nm}$ thick Au layer was deposited on the polished side of the Si wafer using an electron beam evaporator (ULVAC EBX14D). A $5 \mathrm{~nm}$ thick Cr film was deposited as adhesion layer between native silicon oxide and the Au layer.

Semiconductor Deposition: A solution 2 \% wt. of the organic semiconductor 2,8-difluoro5,11-bis(triethylsilylethynyl)-anthradithiophene (diF-TES-ADT) mixed with polystyrene ( $\mathrm{M}_{\mathrm{W}}$ $=10000 \mathrm{gmol}^{-1}$ ) in a 4:1 ratio in chlorobenzene was prepared. The solution was then deposited by the so-termed "Bar-Assisted Meniscus Shearing” on top of the interdigitated source and drain electrodes as previously reported. ${ }^{[35,36]}$

Microfluidic channel: The microfluidic device was fabricated by sticking two parts of doublesided adhesive (each $225 \mu \mathrm{m}$ thick) on top of each other with an intermediate $10 \mu \mathrm{m}$ thick polyethylene terephthalate layer (PET) layer in order to avoid any leakage of aqueous solution outside the chamber. The double-sided adhesive was patterned by means of a laser scan marker "Marko" (Laserpoint SRL, Milan, Italy) which has a pulsed (100 ns width, 20 kHz repetition rate, 50\% duty cycle) Nd:YAG infrared (IR) laser centered at $\lambda=1064 \mathrm{~nm}$. A chamber for the aqueous solution on one side and a smaller window $(2.5 \mathrm{~mm} \times 2.5 \mathrm{~mm})$ on the top side were made, which then served to contact the top gate electrodes with the electrolyte solution. This microfluidic assembly was inserted on top of the source and drain interdigitated electrodes (already covered by OSC). The upper part of the microfluidic chamber bears two well-defined openings which enable a precise positioning of two Au gate electrodes ensuring a proper isolation (discarding any probability of a cross-talk between them) and allowing to contact the electrodes from the top with spring probes. The flow of aqueous analytes is guaranteed by a peristaltic pump (Watson-Marlow 120S/DM2). 
Working Electrode: one of the gold gate electrodes was functionalized by [2-2'-bi(1,3dithiolylidene)]-4-ylmethyl5-(1,2-dithiolan-3-yl)pentanoate $\left(\mathrm{TTFS}_{2}\right)$ following the previously reported methodology. ${ }^{[33]}$ The SAMs were prepared by immersion of the electrodes in a $1 \mathrm{mM}$ solution of $\mathrm{TTFS}_{2}$ in dry THF under an inert atmosphere and room temperature for $72 \mathrm{~h}$.

Electrical Characterization: cyclic voltammetry (CV) measurements have been carried out using a potentiostat/galvanostat 263a (EG\&G Princeton Applied Research). A three-electrode setup was used. The $\mathrm{TTFS}_{2} / \mathrm{Au}$ was employed as the working electrode (WE), while a platinum and a silver wires (0.5 mm of diameter each one) were used as counter (CE) and quasi-reference electrodes (RE), respectively. A solution of $\mathrm{LiClO}_{4}(0.1 \mathrm{M})$ in acetonitrile, or an aqueous solution (0.1 M) of buffer phosphate ( $\mathrm{pH}$ 6.9) and $0.1 \mathrm{M} \mathrm{NaClO}_{4}$ was used as electrolyte. In order to switch between the gate electrodes, a home-made multiplexer was built. The input channel was receiving the gate voltage potential from source-measurement unit (SMU) and delivering in to the output channels where the gate electrodes were connected. An integrated microcontroller with customized written software provided a possibility to select the output channel within a "click of mouse button” time.

All the electrical device measurements were performed with Agilent 5100A SMU by Easy Expert software. The I-V transfer characteristics were recorded at a scan rate of $60 \mathrm{mV} / \mathrm{s}$ with a fixed $V_{D S}$ at $-0.4 \mathrm{~V}$. Prior to oxidation/reduction test, a stability check with both working gate electrode and reference gate electrode were performed.

\section{Acknowledgements}

This work was funded by ITN iSwitch 642196 (V. P. and M. S. M.), ERC StG 2012-306826 e-GAMES, the EuroNanoMed III project “AMI”, the Networking Research Center on Bioengineering, Biomaterials, and Nanomedicine (CIBER-BBN), the Generalitat de Catalunya (2017-SGR-918) and the Spanish Ministry of Economy and Competitiveness, through the project FANCY CTQ2016-80030-R and "Severo Ochoa” Programme for Centers of Excellence in R\&D (SEV-2015-0496). V.P. is enrolled in the Molecular and Regenerative Medicine Programme of the Università di Modena and Reggio Emilia. M. S. M. and Q.Z. are 
enrolled in the Materials Science PhD Program of Universitat Autònoma de Barcelona. S.C. acknowledges the People Programme (Marie Curie Actions) of the Seventh Framework Programme of the European Union (FP7/2007-2013) under Research Executive Agency Grant Agreement No. 600388 (TECNIOSpring programme), and from the Agency for Business Competitiveness of the Government of Catalonia, ACCIÓ. Q. Z. acknowledges the China Scholarship Council, the National Natural Science Foundation (NSF) of China (11404266) and the Fundamental Research Funds for the Central Universities (Grant No. XDJK2011C041).

References

[1] L. Kergoat, L. Herlogsson, D. Braga, B. Piro, M. C. Pham, X. Crispin, M. Berggren, G. Horowitz, Adv. Mater. 2010, 22, 2565.

[2] L. Kergoat, B. Piro, M. Berggren, G. Horowitz, M.-C. Pham, Anal. Bioanal. Chem. 2012, 402, 1813.

[3] K. Melzer, M. Brändlein, B. Popescu, D. Popescu, P. Lugli, G. Scarpa, Faraday Discuss. 2014, 174, 399.

[4] M. Di Lauro, S. Casalini, M. Berto, A. Campana, T. Cramer, M. Murgia, M.

Geoghegan, C. A. Bortolotti, F. Biscarini, ACS Appl. Mater. Interfaces 2016, 8, 31783.

[5] T. Cramer, A. Kyndiah, M. Murgia, F. Leonardi, S. Casalini, F. Biscarini, Appl. Phys. Lett. 2012, 100, 2010.

[6] D. Wang, V. Noël, B. Piro, Electronics 2016, 5, 9.

[7] L. Kergoat, B. Piro, M. Berggren, M. C. Pham, A. Yassar, G. Horowitz, Org. Electron. physics, Mater. Appl. 2012, 13, 1.

[8] M. Y. Mulla, E. Tuccori, M. Magliulo, G. Lattanzi, G. Palazzo, K. Persaud, L. Torsi, Nat. Commun. 2015, 6, 1.

[9] S. Casalini, F. Leonardi, T. Cramer, F. Biscarini, Org. Electron. physics, Mater. Appl. 2013, 14, 156.

[10] T. Cramer, B. Chelli, M. Murgia, M. Barbalinardo, E. Bystrenova, D. M. de Leeuw, F. 
Biscarini, Phys. Chem. Chem. Phys. 2013, 15, 3897.

[11] S. Casalini, A. C. Dumitru, F. Leonardi, C. A. Bortolotti, E. T. Herruzo, A. Campana, R. F. de Oliveira, T. Cramer, R. Garcia, F. Biscarini, ACS Nano 2015, 9, 5051.

[12] M. Berto, C. Diacci, R. D’Agata, M. Pinti, E. Bianchini, M. Di Lauro, S. Casalini, A. Cossarizza, M. Berggren, D. Simon, G. Spoto, F. Biscarini, C. A. Bortolotti, Adv. Biosyst. 2017, 1700072, 1700072.

[13] C. Diacci, M. Berto, M. Di Lauro, E. Bianchini, M. Pinti, D. T. Simon, F. Biscarini, C. A. Bortolotti, Biointerphases 2017, 12, 05F401.

[14] M. Berto, S. Casalini, M. Di Lauro, S. L. Marasso, M. Cocuzza, D. Perrone, M. Pinti, A. Cossarizza, C. F. Pirri, D. T. Simon, M. Berggren, F. Zerbetto, C. A. Bortolotti, F. Biscarini, Anal. Chem. 2016, 88, 12330.

[15] H. Klauk, U. Zschieschang, J. Pflaum, M. Halik, Nature 2007, 445, 745.

[16] E. J. Meijer, D. M. De Leeuw, S. Setayesh, E. Van Veenendaal, B. H. Huisman, P. W. M. Blom, J. C. Hummelen, U. Scherf, T. M. Klapwijk, Nat. Mater. 2003, 2, 678.

[17] Q. J. Sun, J. Peng, W. H. Chen, X. J. She, J. Liu, X. Gao, W. L. Ma, S. D. Wang, Org. Electron. physics, Mater. Appl. 2016, 34, 118.

[18] Z. Zhang, X. Ren, B. Peng, Z. Wang, X. Wang, K. Pei, B. Shan, Q. Miao, P. K. L. Chan, Adv. Funct. Mater. 2015, 25, 6112.

[19] D. Khim, H. Han, K. J. Baeg, J. Kim, S. W. Kwak, D. Y. Kim, Y. Y. Noh, Adv. Mater. 2013, 25, 4302.

[20] W. Smaal, C. Kjellander, Y. Jeong, A. Tripathi, B. Van Der Putten, A. Facchetti, H. Yan, J. Quinn, J. Anthony, K. Myny, W. Dehaene, G. Gelinck, Org. Electron. physics, Mater. Appl. 2012, 13, 1686.

[21] Y.-H. Chou, H.-C. Chang, C.-L. Liu, W.-C. Chen, Polym. Chem. 2015, 6, 341.

[22] M. Yi, J. Shu, Y. Wang, H. Ling, C. Song, W. Li, L. Xie, W. Huang, Org. Electron. physics, Mater. Appl. 2016, 33, 95. 
[23] T. Leydecker, M. Herder, E. Pavlica, G. Bratina, S. Hecht, E. Orgiu, P. Samorì, Nat. Nanotechnol. 2016, 11, 769.

[24] B. Yaman, I. Terkesli, K. M. Turksoy, A. Sanyal, S. Mutlu, Org. Electron. physics, Mater. Appl. 2014, 15, 646.

[25] R. Porrazzo, A. Luzio, S. Bellani, G. E. Bonacchini, Y. Y. Noh, Y. H. Kim, G.

Lanzani, M. R. Antognazza, M. Caironi, ACS Omega 2017, 2, DOI

10.1021/acsomega.6b00256.

[26] M. Giordani, M. Di Lauro, M. Berto, C. A. Bortolotti, D. Vuillaume, H. L. Gomes, M. Zoli, F. Biscarini, 2016, p. 99440P-9944-7.

[27] Y. Van De Burgt, E. Lubberman, E. J. Fuller, S. T. Keene, G. C. Faria, S. Agarwal, M. J. Marinella, A. Alec Talin, A. Salleo, Nat. Mater. 2017, 16, 414.

[28] E. Juzekaeva, A. Nasretdinov, S. Battistoni, T. Berzina, S. Iannotta, R. Khazipov, V. Erokhin, M. Mukhtarov, Adv. Mater. Technol. 2018, 2, 1800350.

[29] M. Giordani, M. Berto, M. Di Lauro, C. A. Bortolotti, M. Zoli, F. Biscarini, ACS Sensors 2017, 2, 1756.

[30] Z. Liu, A. A. Yasseri, J. S. Lindsey, D. F. Bocian, Science (80-. ). 2003, 302, 1543.

[31] M. Mas-Torrent, C. Rovira, J. Veciana, Adv. Mater. 2013, 25, 462.

[32] E. Marchante, N. Crivillers, M. Buhl, J. Veciana, M. Mas-Torrent, Angew. Chemie Int. Ed. 2016, 55, 368.

[33] C. Simão, M. Mas-Torrent, N. Crivillers, V. Lloveras, J. M. Artés, P. Gorostiza, J. Veciana, C. Rovira, Nat. Chem. 2011, 3, 359.

[34] M. Burkhardt, A. Jedaa, M. Novak, A. Ebel, K. Voïtchovsky, F. Stellacci, A. Hirsch, M. Halik, Adv. Mater. 2010, 22, 2525.

[35] I. Temiño, F. G. Del Pozo, M. R. Ajayakumar, S. Galindo, J. Puigdollers, M. MasTorrent, Adv. Mater. Technol. 2016, 1, 1600090.

[36] Q. Zhang, F. Leonardi, S. Casalini, I. Temiño, M. Mas-Torrent, Sci. Rep. 2016, 6, 
39623.

[37] E. Marchante, M. S. Maglione, N. Crivillers, C. Rovira, M. Mas-Torrent, RSC Adv. 2017, 7, 5636.

[38] S. Casalini, C. A. Bortolotti, F. Leonardi, F. Biscarini, Chem. Soc. Rev. 2017, 46, 40.

[39] L. Kergoat, L. Herlogsson, B. Piro, M. C. Pham, G. Horowitz, X. Crispin, M. Berggren, Proc. Natl. Acad. Sci. U. S. A. 2012, 109, 8394.

[40] S. P. White, K. D. Dorfman, C. D. Frisbie, J. Phys. Chem. C 2016, 120, 108.

[41] M. J. Spijkman, J. J. Brondijk, T. C. T. Geuns, E. C. P. Smits, T. Cramer, F. Zerbetto, P. Stoliar, F. Biscarini, P. W. M. Blom, D. M. De Leeuw, Adv. Funct. Mater. 2010, 20, 898.

[42] Y. M. Park, A. Salleo, Appl. Phys. Lett. 2009, 95, 2007.

[43] T. Xu, L. Xiang, M. Xu, W. Xie, W. Wang, Sci. Rep. 2017, 7, 1.

[44] M. Xu, S. Guo, T. Xu, W. Xie, W. Wang, Org. Electron. physics, Mater. Appl. 2019, 64, 62.

[45] M. S. Lu, H. C. Wu, Y. W. Lin, M. Ueda, W. C. Chen, React. Funct. Polym. 2016, 108, 39.

[46] W. Li, F. Guo, H. Ling, H. Liu, M. Yi, P. Zhang, W. Wang, L. Xie, W. Huang, Small 2018, $14,1$.

[47] P. Gkoupidenis, N. Schaefer, B. Garlan, G. G. Malliaras, Adv. Mater. 2015, 27, 7176.

[48] Q. Lai, L. Zhang, Z. Li, W. F. Stickle, R. S. Williams, Y. Chen, Adv. Mater. 2010, 22, 2448.

[49] P. Gkoupidenis, N. Schaefer, X. Strakosas, J. A. Fairfield, G. G. Malliaras, Appl. Phys. Lett. 2015, 107, DOI 10.1063/1.4938553.

[50] L. an Kong, J. Sun, C. Qian, Y. Fu, J. Wang, J. Yang, Y. Gao, Org. Electron. physics, Mater. Appl. 2017, 47, 126.

[51] C. Qian, J. Sun, L. A. Kong, G. Gou, J. Yang, J. He, Y. Gao, Q. Wan, ACS Appl. 
Mater. Interfaces 2016, 8, 26169.

[52] G. Liu, C. Wang, W. Zhang, L. Pan, C. Zhang, X. Yang, F. Fan, Y. Chen, R. W. Li, Adv. Electron. Mater. 2016, 2, 1.

[53] L. McCay-Peet, E. Toms, Inf. Res. 2011, 16, 1.

[54] F. Alibart, S. Pieutin, D. Guérin, C. Novembre, S. Lenfant, K. Lmimouni, C. Gamrat, D. Vuillaume, Adv. Funct. Mater. 2010, 20, 330. 
